\title{
Supporting Information \\ Lyotropic Chromonic Liquid Crystals and Their Impurities Reveal the Importance of the Position of Functional Groups in Self-Assembly
}

\author{
Jonghee Eun, Jiyong Cheon, Sung-Jo Kim, Tae Joo Shin, Joonwoo Jeong
}

Comparison of the Area Fractions of Peaks in Liquid Chromatogram of SSY Before and After the Purification

\begin{tabular}{cccc}
\hline Peak No. & $\begin{array}{c}\text { Nonpurified SSY } \\
{[\%]}\end{array}$ & $\begin{array}{c}\text { Purified SSY } \\
{[\%]}\end{array}$ & Identified impurities \\
\hline 1 & 3.63 & 4.09 & RS-SA or GS-SA \\
2 & 0.54 & 0.33 & Sub A \\
3 & 94.75 & 95.52 & SSY \\
4 & 0.17 & - & DONS \\
5 & 0.46 & - & 2 N-SA or SS-AN \\
\hline
\end{tabular}

Table S1: The first column indicates the peak number in Figure 3 of the main text. The area fraction is shown in \%. The rightmost column shows the identified impurities in each chromatogram peak. Note the fourth and fifth peaks are not detected after the purification. On the contrary, the area fraction of the peak \#3 containing the SSY increases slightly after the purification as well as the peak \#1 containing RS-SA or GS-SA. The caveat is that the increment does not necessarily mean the increase in the amount of SSY and trivalent impurities such as GS-SA or RS-SA. It is because each peak contains other unidentified chemicals as shown in Figures S1 and S2. Especially, RS-SA or GS-SA in the peak \#1 is one of the minor species, whereas SSY in the peak \#3 is the dominant one. 

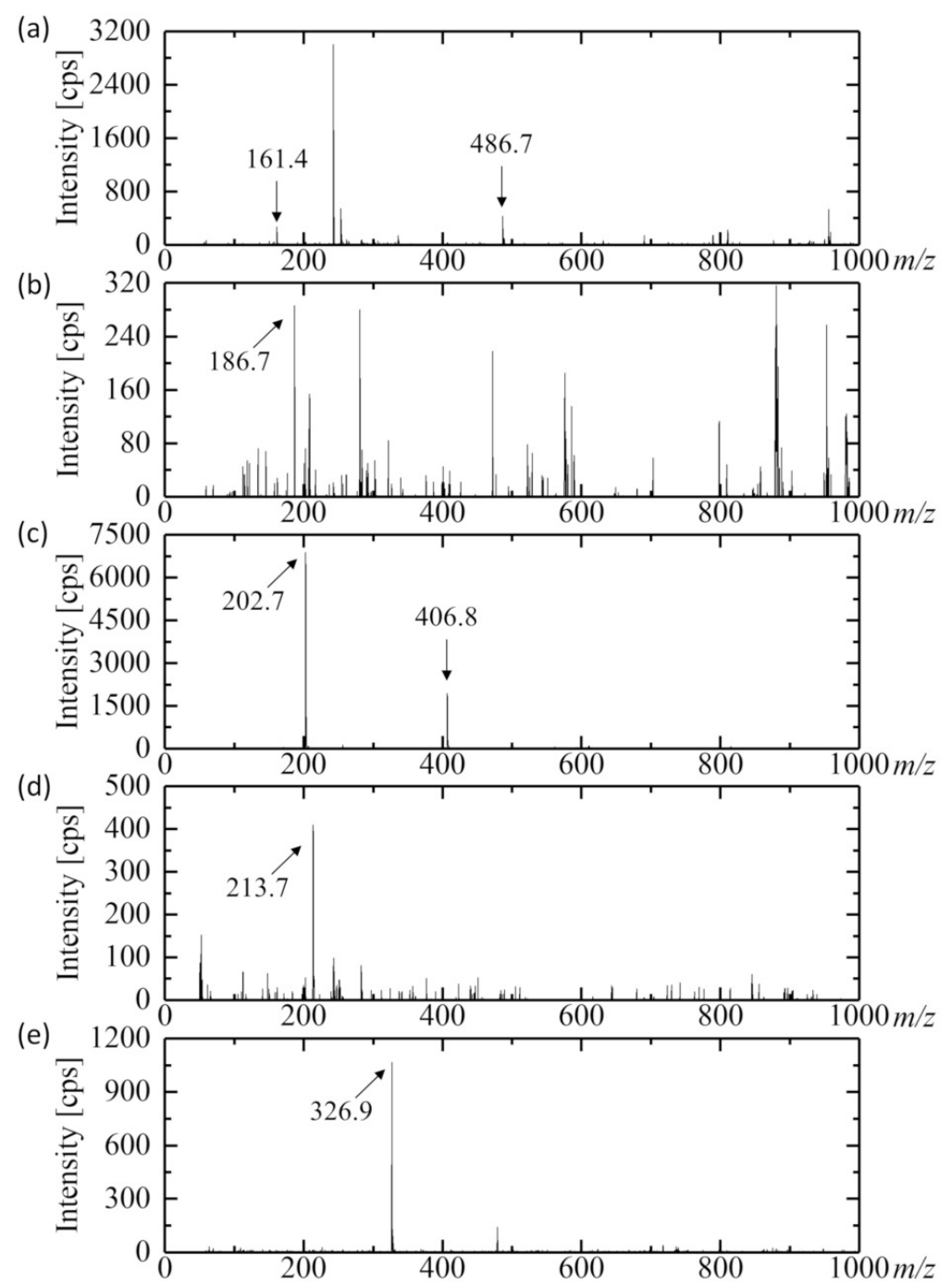

Figure S1: Mass spectra of SSY before the purification. (a-e) represent respectively the mass spectrum of each peak from \#1 to \#5 in Figure 3a of the main text. The identified mass-to-charge ratios $m / z$ are indicated with their multiples. 


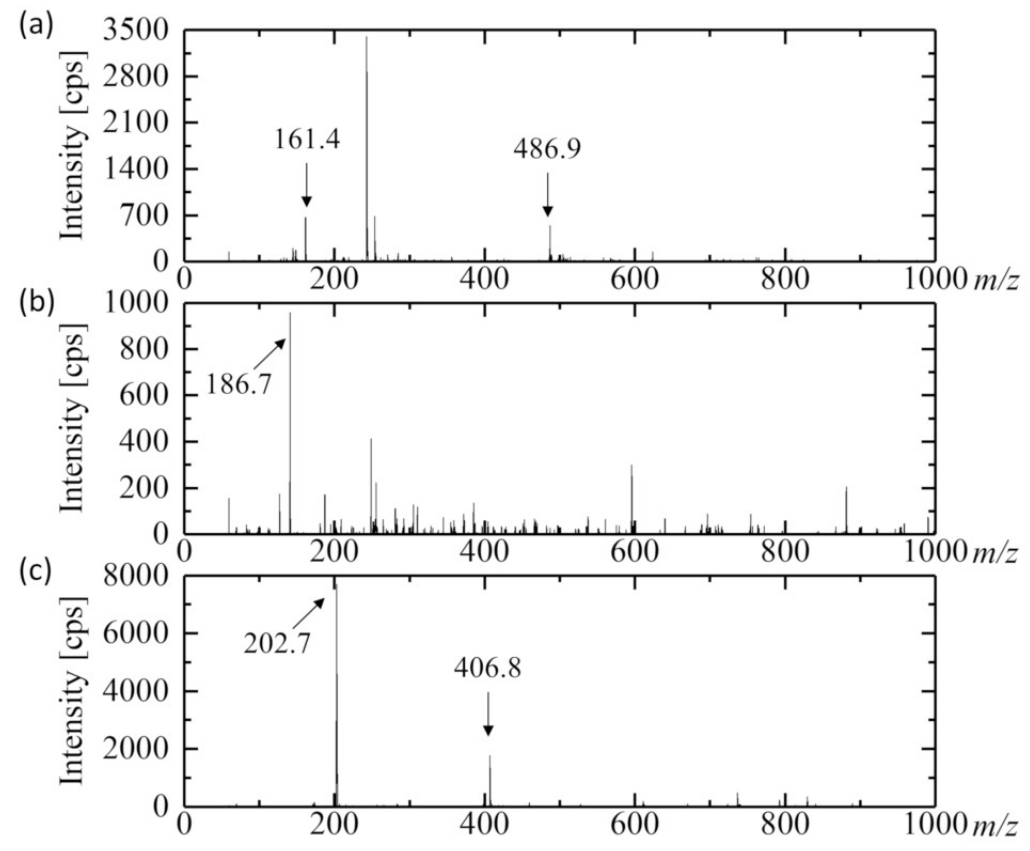

Figure S2: Mass spectra of SSY after the purification. (a-e) represent respectively the mass spectrum of each peak from \#1 to \#3 in Figure 3b of the main text. The identified mass-to-charge ratios $m / z$ are indicated with their multiples. 

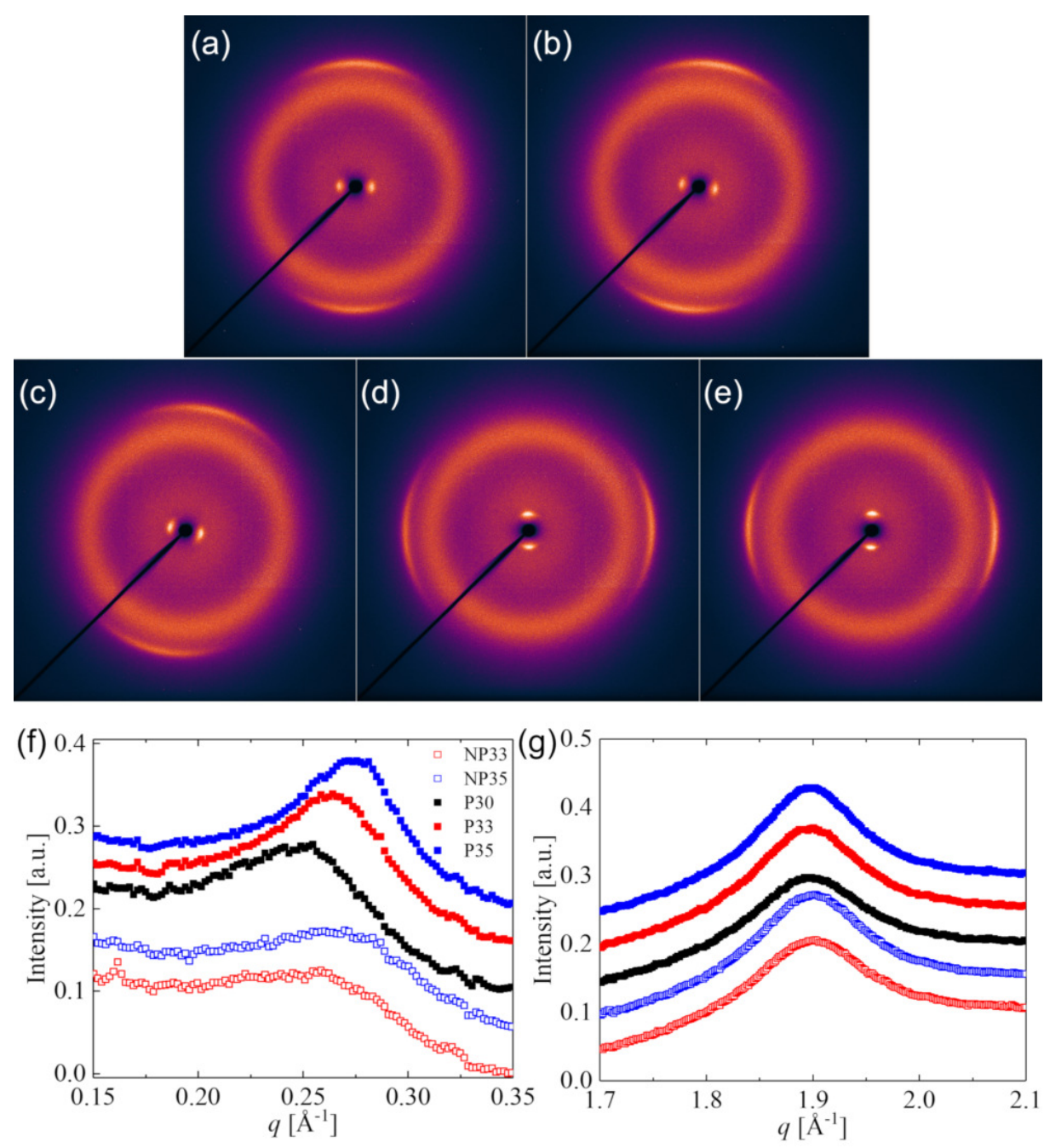

Figure S3: Comparison of the X-ray scattering data of SSY before and after the purification according to the concentration. (a-e) The two dimensional wide-angle X-ray diffraction (WAXD) patterns of SSY. (a) and (b) The 2D WAXD patterns (the top row) of non-purified SSY of 33.0 and $35.0 \%$ (wt/wt), respectively. (c-e) The 2D WAXD patterns (the bottom row) of purified SSY of 30.0, 33.0, and 35.0\% (wt/wt), respectively. (f) and (g) The angle averaged intensity profiles of the small-angle and wide-angle regimes of (a-e), respectively. Each intensity data is angle-averaged, background-subtracted, and offset by 0.05 for clear presentation. NP33 and NP35 shown with open symbols are the data from the Nonpurified SSY of the concentration 33.0 and $35.0 \%$ (wt/wt), respectively. The filled symbols are from the purified SSY of different concentrations, i.e., 30.0, 33.0, and $35.0 \%$ (wt/wt). All X-ray experiments are conducted at $26.0^{\circ} \mathrm{C}$. 

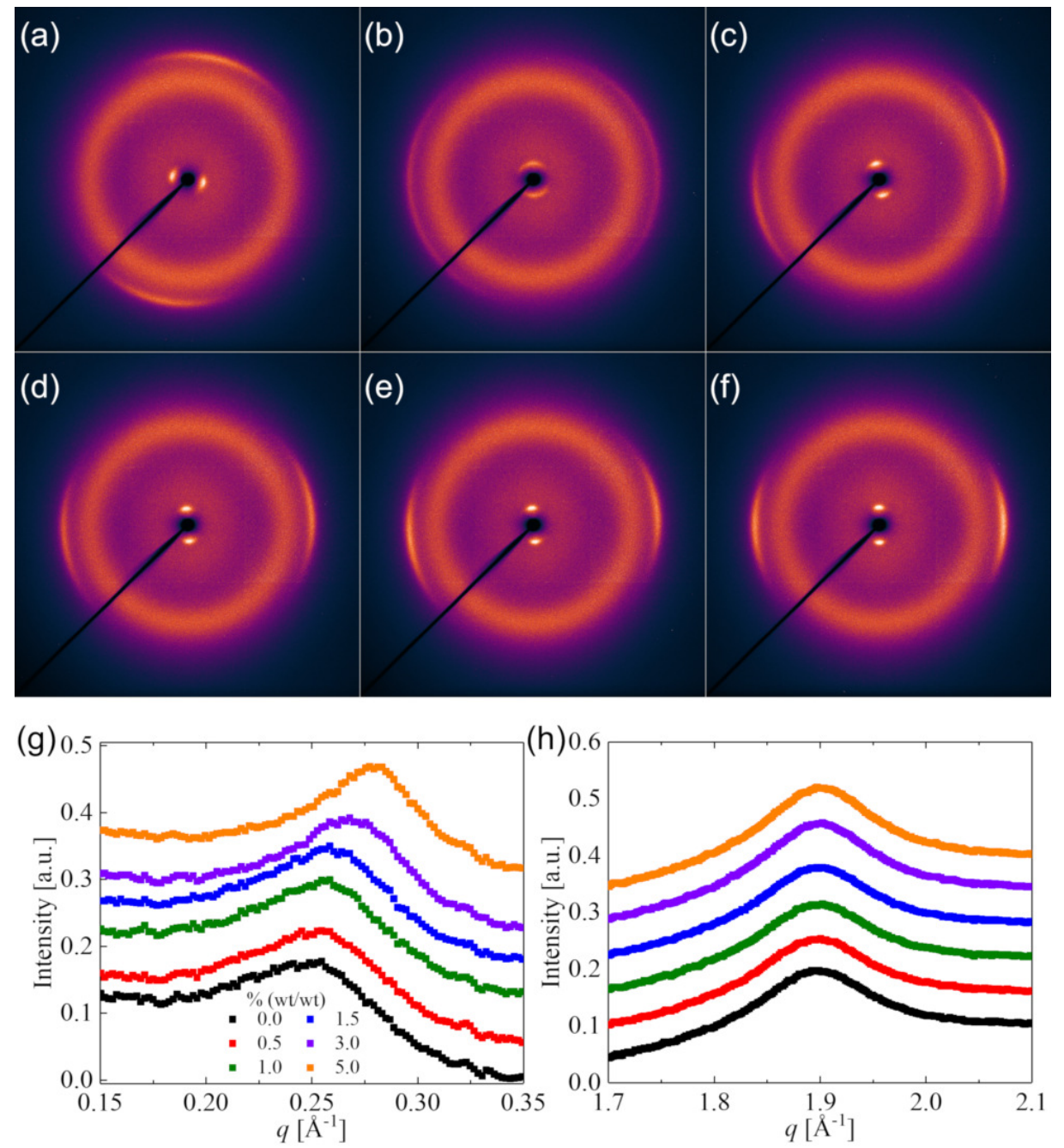

Figure S4: Comparison of the X-ray scattering data of purified $30.0 \%$ (wt/wt) nematic SSY according to the different concentration of the added 2N-SA from 0.0 to $5.0 \%$ (wt/wt). (a-e) The two dimensional wide-angle X-ray diffraction patterns of SSY with the different concentration of $2 \mathrm{~N}-\mathrm{SA}$ doped. The concentrations of $2 \mathrm{~N}-\mathrm{SS}$ are (a) 0.0, (b) 0.5, (c) 1.0, (d) 1.5, (e) 3.0, and (f) $5.0 \%$ (wt/wt), respectively. (g) and $(\mathrm{h})$ The angle averaged intensity profiles of the small-angle and wideangle regimes of (a-f), respectively. Each intensity data is angle-averaged, background-subtracted, and offset by 0.06 for clear presentation. All X-ray experiments are conducted at $26.0^{\circ} \mathrm{C}$. 

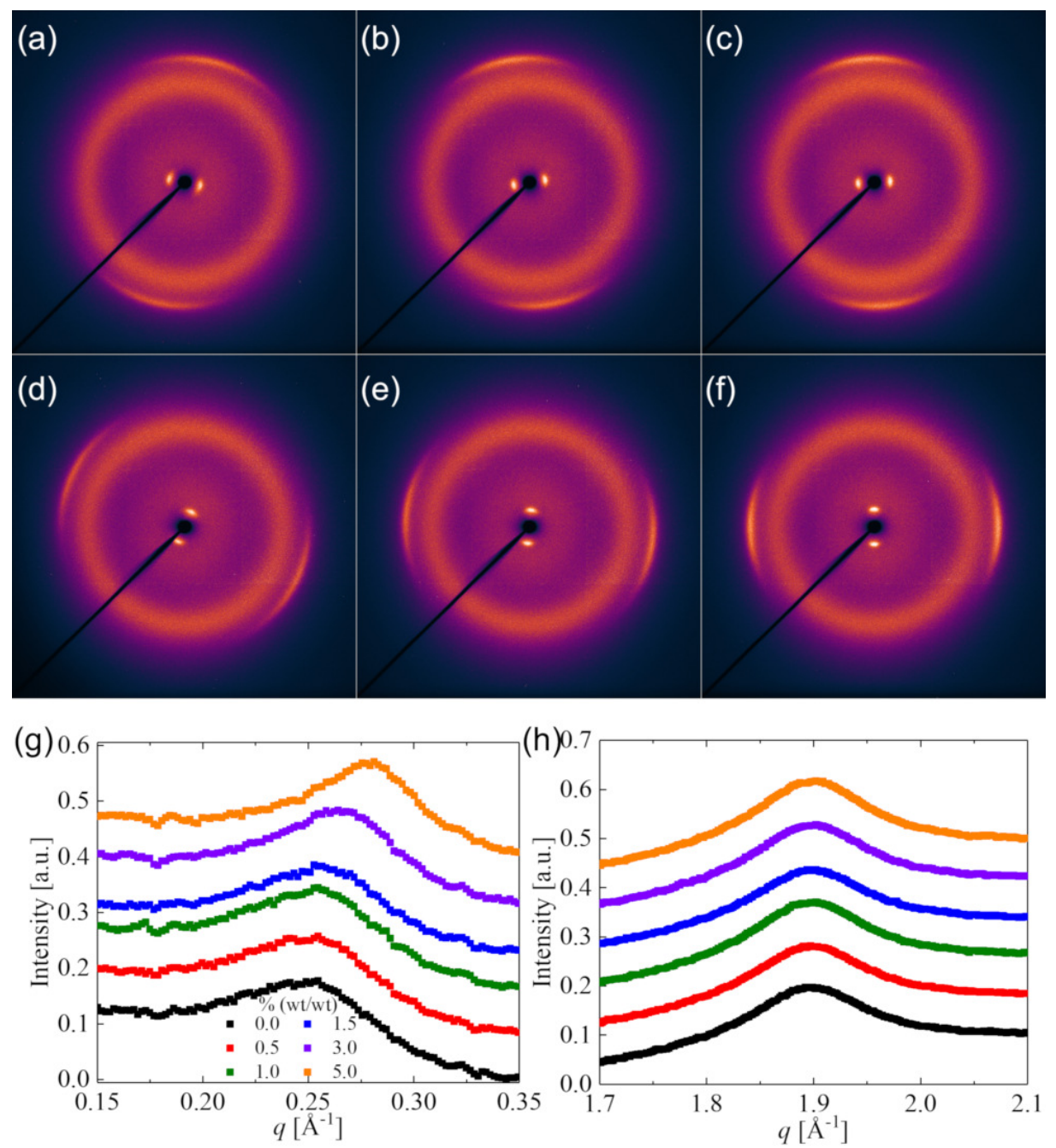

Figure S5: Comparison of the X-ray scattering data of purified $30.0 \%$ (wt/wt) nematic SSY according to the different concentration of the added SS-AN from 0.0 to $5.0 \%$ (wt/wt). (a-e) The two dimensional wide-angle X-ray diffraction patterns of SSY with the different concentration of SS-SA doped. The concentrations of SS-SA are (a) 0.0, (b) 0.5, (c) 1.0, (d) 1.5, (e) 3.0, and (f) $5.0 \%$ (wt/wt), respectively. (g) and $(\mathrm{h})$ The angle averaged intensity profiles of the small-angle and wideangle regimes of (a-f), respectively. Each intensity data is angle-averaged, background-subtracted, and offset by 0.08 for clear presentation. All X-ray experiments are conducted at $26.0^{\circ} \mathrm{C}$. 

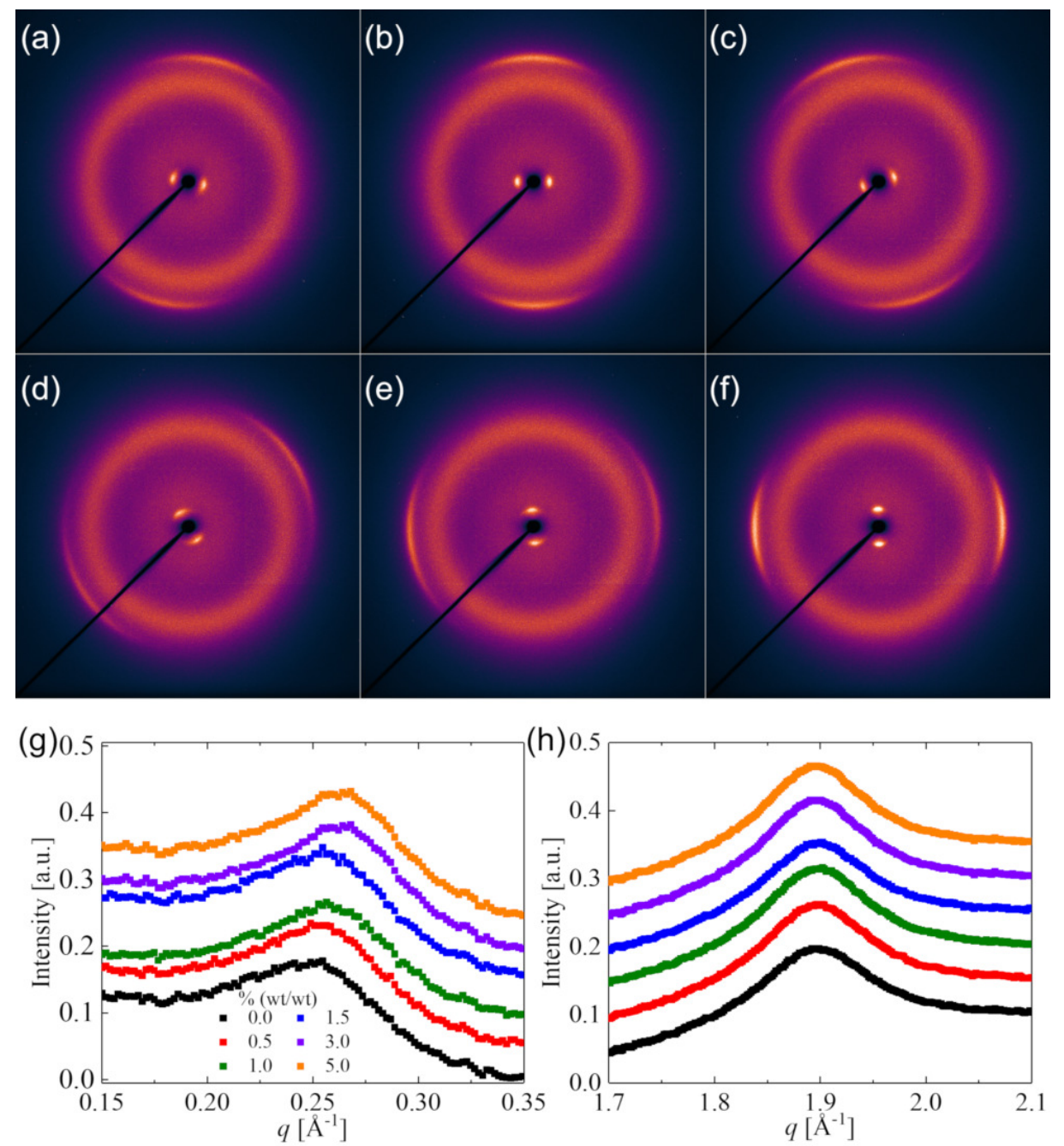

Figure S6: Comparison of the X-ray scattering data of purified $30.0 \%$ (wt/wt) nematic SSY according to the different concentration of the added RS-SA from 0.0 to $5.0 \%$ (wt/wt). (a-e) The two dimensional wide-angle X-ray diffraction patterns of SSY with the different concentration of RS-SA doped. The concentrations of RS-SA are (a) 0.0, (b) 0.5, (c) 1.0, (d) 1.5, (e) 3.0, and (f) $5.0 \%$ (wt/wt), respectively. (g) and $(\mathrm{h})$ The angle averaged intensity profiles of the small-angle and wideangle regimes of (a-f), respectively. Each intensity data is angle-averaged, background-subtracted, and offset by 0.05 for clear presentation. All X-ray experiments are conducted at $26.0^{\circ} \mathrm{C}$. 

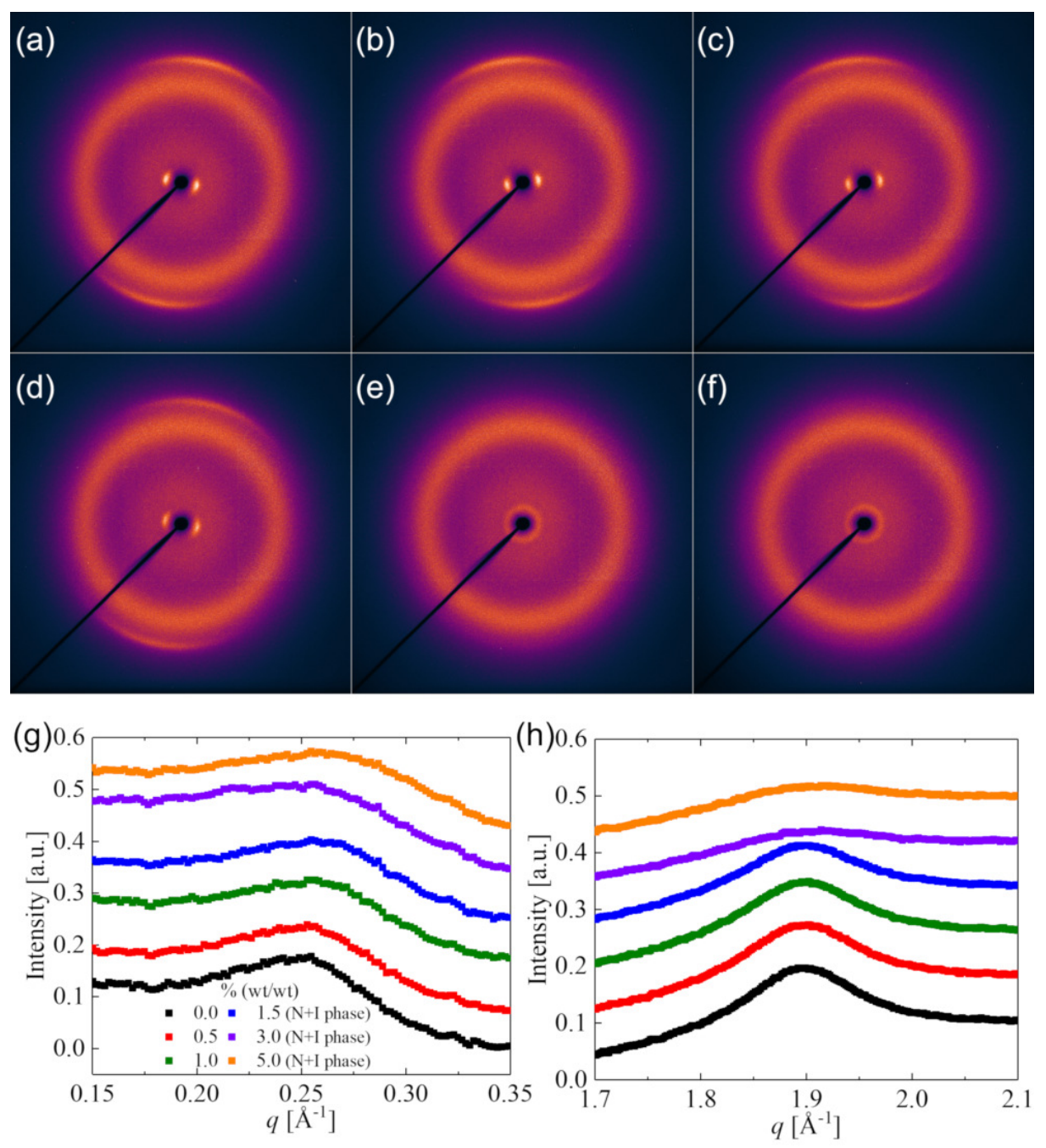

Figure S7: Comparison of the X-ray scattering data of purified 30.0\% (wt/wt) SSY according to the different concentration of the added GS-SA from 0.0 to $5.0 \%$ (wt/wt). (a-e) The two dimensional wide-angle X-ray diffraction patterns of SSY with the different concentration of GS-SA doped. The concentrations of GS-SA are (a) 0.0, (b) 0.5, (c) 1.0, (d) 1.5, (e) 3.0, and (f) $5.0 \%$ (wt/wt), respectively. The angle averaged intensity profiles of the small-angle and wide-angle regimes of (af), respectively. Each intensity data is angle-averaged, background-subtracted, and offset by 0.08 for clear presentation. Note that the SSY has the nematic-isotropic coexistence phase when the concentration of GS-SA surpasses $1.5 \%$ (wt/wt). All X-ray experiments are conducted at $26.0^{\circ} \mathrm{C}$. 


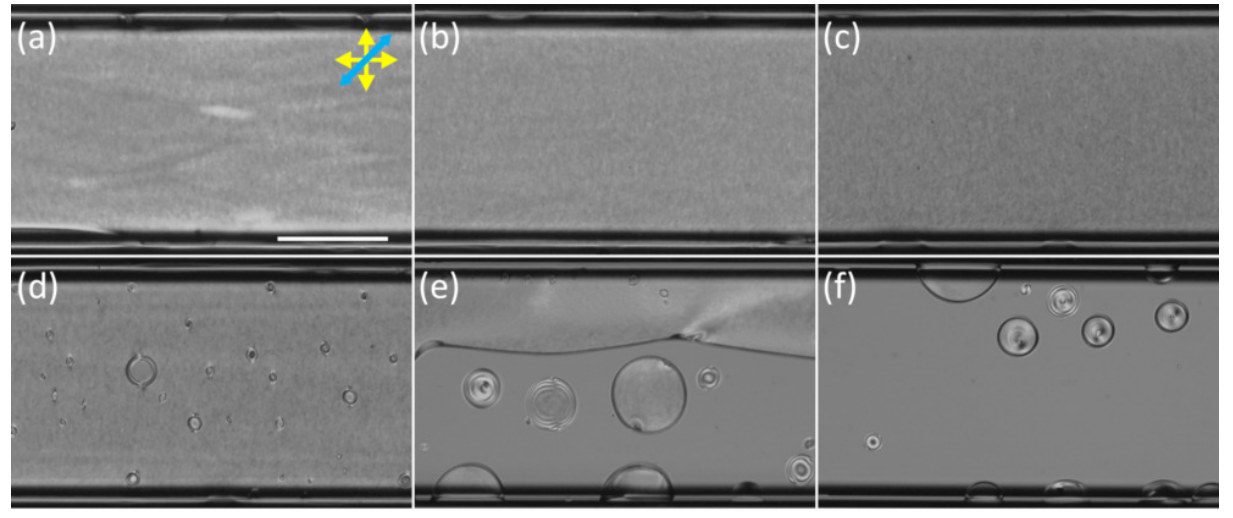

Figure S8: Polarized optical microscopy images of the $30.0 \%$ (wt/wt) SSY according to the different concentration of the added GS-SA. The concentrations of the added GS-SA are (a) 0.0, (b) 0.5, (c) 1.0, (d) 1.5 , (e) 3.0, and (f) $5.0 \%$ (wt/wt), respectively. All samples are confined in rectangular capillaries with the optical path length of $50 \mu \mathrm{m}$, and the temperature is $21.5^{\circ} \mathrm{C}$. The scale bar is $250 \mu \mathrm{m}$. The sample is placed between crossed polarizers, i.e., polarizer and analyzer, and a full wave plate of 550-nm retardance is inserted before the analyzer. The yellow double arrows represent the pass axes of crossed polarizers, and blue arrow represents the slow axis of the full wave plate. The SSY in (a-c) exhibits the fully nematic phase. As the concentration of GS-SA exceeds $1.5 \%$ (wt/wt), isotropic droplets in the nematic phase start to appear in (d). As shown in (d-f), the isotropic phase in the coexistence phase becomes dominant as the concentration of the GS-SA increases; the spherical droplets in (f) are nematic droplets dispersed in the isotropic phase. 
(a)

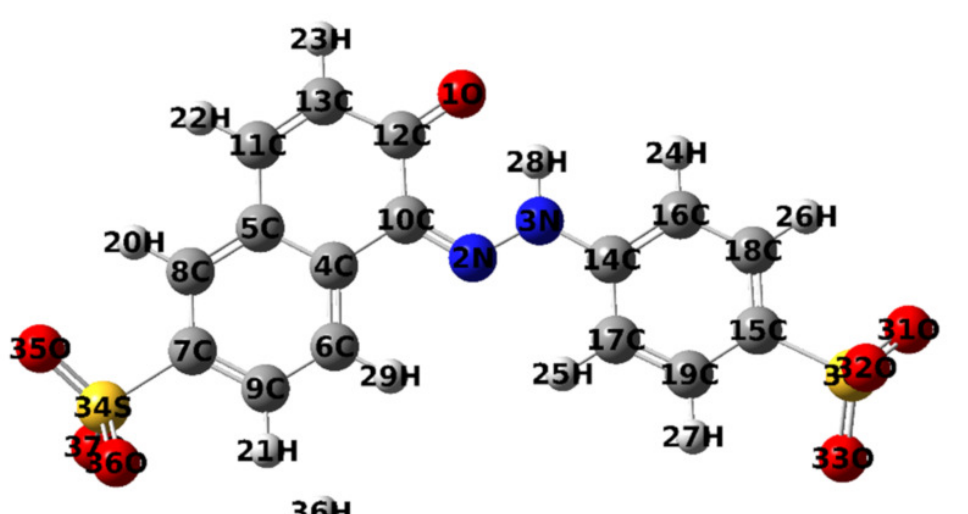

(b)

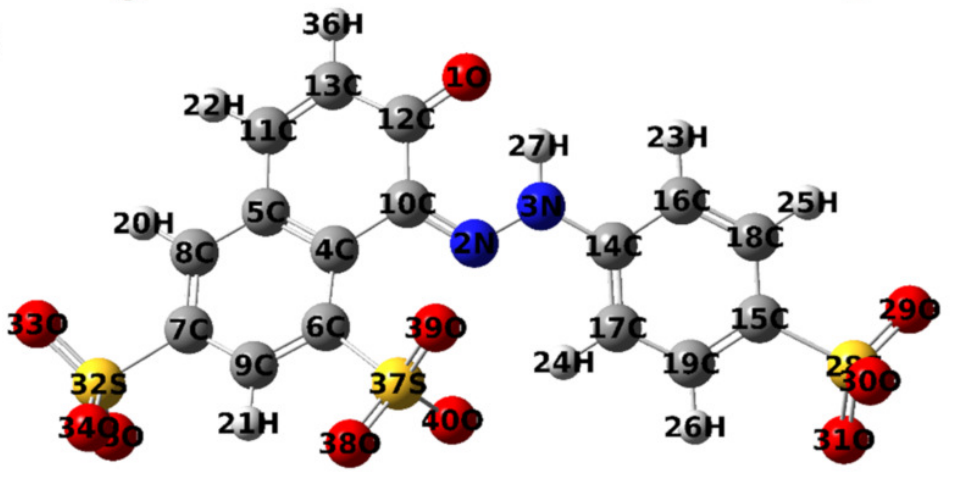

(c)

\begin{tabular}{ccc}
\hline $\begin{array}{c}\text { Dihedral angles } \\
\text { [degree] }\end{array}$ & SSY & GS-SA \\
\hline 14C-3N-2N-10C & 179.57 & -174.23 \\
3N-2N-10C-4C & 179.98 & -178.43 \\
2N-3N-14C-17C & 0.29 & -0.62 \\
2N-10C-4C-6C & -0.04 & 20.09 \\
16C-14C-17C-19C & -0.37 & 0.30 \\
5C-4C-6C-9C & 0.14 & 6.11 \\
10C-4C-5C-11C & -0.05 & -6.32 \\
\hline
\end{tabular}

Figure S9: Optimized molecular structures of SSY and GS-SA in NH hydrazone forms with the atom labels. Top view of the calculated 3D structure of (a) SSY and (b) GS-SA. (c) Comparison of the selected dihedral angles of SSY and GS-SA in (a) and (b), respectively. 